\title{
A NOTE ON THE FALKNER-SKAN FLOWS OF A NON-NEWTONIAN FLUID
}

\author{
K. R. RAJAGOPAL \\ Department of Mechanical Engineering, University of Pittsburgh, Pittsburgh, PA 15261, U.S.A.
}

\author{
A. S. GuPtA
}

Department of Mathematics, Indian Institute of Technology, Kharagpar, 721302, India

and

T. Y. NA

Department of Mechanical Engincering. The University of Michigan, Dearborn, MI 48128, U.S.A.

(Receiced 20 October 1982)

\begin{abstract}
Non-similar solutions are established for the boundary layer flow of a homogeneous incompressible fluid of second grade past a wedge placed symmetrically with respect to the flow direction. The variation of the skin-friction with respect to the non-Newtonian parameters is discussed.
\end{abstract}

\section{INTRODUCTION}

In recent years there has been some interest in the boundary layer flows of non-Newtonian fluids. Srivatsava [1] and Rajeswari and Rathna [2] investigated boundary layer flows of the incompressible Rivlin-Eriksen fluid of second order for the stagnation flow problems. Beard and Walters [3] studied boundary layer flows of more general elastico-viscous fluids by employing a perturbation analysis. Recently, Rajagopal et al. [4] have looked at the boundary layer flows of fluids of second grade and they point out that care has to be exercised in such analysis which could otherwise be fraught with inherent inconsistencies.

In this paper we study the Falkner-Skan flows of a homogeneous incompressible fluid of second grade, i.e., the flow past a wedge placed symmetrically with respect to the flow direction. The Cauchy stress $T$ in a fluid of second grade is related to the fluid motion in the following manner (cf. Truesdell and Noll [5])

$$
\mathbf{T}=-p \mathbf{1}+\mu \mathbf{A}_{1}+\alpha_{1} \mathbf{A}_{2}+\alpha_{2} \mathbf{A}_{1}^{2}
$$

where $p$ is the pressure, $\mu$ the coefficient of viscosity and $\alpha_{1}$ and $\alpha_{2}$ material moduli which are usually referred to as the normal stress coefficients. The kinematical tensors $\mathbf{A}_{1}$ and $\mathbf{A}_{2}$ are defined through (cf. Rivlin and Ericksen [6])

$$
\begin{gathered}
\mathbf{A}_{1}=\operatorname{grad} \mathbf{v}+(\operatorname{grad} \mathbf{v})^{T}, \\
\mathbf{A}_{2}=\frac{\mathrm{d}}{\mathrm{d} t} \mathbf{A}_{1}+\mathbf{A}_{1}(\operatorname{grad} \mathbf{v})+\left(\operatorname{grad} \mathbf{v}^{T}\right) \mathbf{A}_{1}
\end{gathered}
$$

where $\mathrm{r}$ denotes the velocity and $\frac{\mathrm{d}}{\mathrm{d} t}$ the material time derivative.

The model (1) has been the object of detailed study in recent years. While the model (1) can be considered as a second order approximation of a simple fluid in the sense of retardation (cf. Coleman and Noll [7]), since the relation (1) is properly invariant it has also been employed as an exact model for some fluids. An analysis based on the assumption that the model is exact and is compatible with thermodynamics in the sense that all motions of the fluid meet 
the Clausius-Duhem inequality and the assumption that the specific Helmholtz free energy be a minimum in equilibrium yields (cf. Dunn and Fosdick [8])

$$
\mu \geq 0, \alpha_{1} \geq 0 \text { and } \alpha_{1}+\alpha_{2}=0 .
$$

In this paper we shall assume that (1) is a model in the sense of a second order approximation.

\section{BOUNDARY LAYER EQUATIONS}

We now proceed to derive the boundary layer equations for the plane flow of an incompressible fluid of second grade. Substituting (1) into the balance of linear momentum

$$
\operatorname{div} \mathbf{T}+\rho \mathbf{b}=\rho \frac{\mathrm{d} \mathbf{v}}{\mathrm{d} t},
$$

and making use of the fact that the fluid can undergo only isochoric motion since it is incompressible, i.e.,

$$
\operatorname{div} \mathbf{v}=0,
$$

we obtain the equation of motion

$$
\begin{aligned}
& \mu \Delta \mathbf{v}+\alpha_{1} \Delta \mathbf{v}_{t}+\alpha_{1}(\Delta \mathbf{w} x \mathbf{v})+\left(\alpha_{1}+\alpha_{2}\right)\left\{\mathbf{A}_{1} \Delta \mathbf{v}+2 \operatorname{div}\left([\operatorname{grad} \mathbf{v}][\operatorname{grad} \mathbf{v}]^{T}\right)\right\} \\
& -\rho \mathbf{v}_{t}-\rho \mathbf{w} x \mathbf{v}=\operatorname{grad} P
\end{aligned}
$$

where

and

$$
\begin{gathered}
\mathbf{w}=\operatorname{curl} \mathbf{v}, \\
P=p-\alpha_{1} v \cdot \Delta \mathbf{v}-\left(2 \alpha_{1}+\alpha_{2}\right)\left|\mathbf{A}_{1}\right|^{2}+\frac{1}{2} \rho|\mathbf{v}|^{2}+\rho \phi .
\end{gathered}
$$

We have assumed that the body force $b$ is conservative, i.e., $b=\operatorname{grad} \phi$. Also, $\Delta$ denotes the Laplacian, $\mathbf{v}_{t}$ denotes the partial derivative of $\mathbf{v}$ with respect to time, $|\mathbf{v}|$ and $\left|A_{1}\right|$ the usual norm of a vector $\mathbf{v}$ and the trace norm of $\mathbf{A}_{1}$ respectively.

In the case of steady plane flow, equation (6) reduces to

$$
\begin{aligned}
u \frac{\partial u}{\partial x}+r \frac{\partial u}{\partial y}= & -\frac{1}{\rho} \frac{\partial p}{\partial x}+\frac{\mu}{\rho}\left(\frac{\partial^{2} u}{\partial x^{2}}+\frac{\partial^{2} u}{\partial y^{2}}\right)+\frac{\alpha_{1}}{\rho}\left\{2 \frac{\partial^{2}}{\partial x^{2}}\left(u \frac{\partial u}{\partial x}+v \frac{\partial u}{\partial y}\right)\right. \\
& +4\left(\frac{\partial u}{\partial x} \frac{\partial^{2} u}{\partial x^{2}}+\frac{\partial v}{\partial x} \frac{\partial^{2} u}{\partial x^{2}}\right)+\frac{\partial^{2}}{\partial x \partial y}\left(u \frac{\partial v}{\partial x}+v \frac{\partial v}{\partial y}\right)+\frac{\partial^{2}}{\partial y^{2}}\left(u \frac{\partial u}{\partial x}+v \frac{\partial u}{\partial y}\right) \\
& \left.+2 \frac{\partial}{\partial y}\left(\frac{\partial u}{\partial x} \frac{\partial u}{\partial y}+\frac{\partial v}{\partial x} \frac{\partial v}{\partial y}\right)\right\}, \\
u \frac{\partial v}{\partial x}+r \frac{\partial v}{\partial y}= & -\frac{1}{\rho} \frac{\partial p}{\partial y}+\mu\left(\frac{\partial^{2} v}{\partial x^{2}}+\frac{\partial^{2} v}{\partial y^{2}}\right)+\frac{\alpha_{1}}{\rho}\left\{2 \frac{\partial^{2}}{\partial y^{2}}\left(u \frac{\partial v}{\partial x}+v \frac{\partial v}{\partial y}\right)\right. \\
& +4\left(\frac{\partial u}{\partial y} \frac{\partial^{2} u}{\partial y^{2}}+\frac{\partial v}{\partial y} \frac{\partial^{2} v}{\partial y^{2}}\right)+\frac{\partial^{2}}{\partial x \partial y}\left(u \frac{\partial u}{\partial x}+v \frac{\partial u}{\partial y}\right) \\
& \left.+\frac{\partial^{2}}{\partial x^{2}}\left(u \frac{\partial v}{\partial x}+v \frac{\partial v}{\partial y}\right)+2 \frac{\partial}{\partial x}\left(\frac{\partial u}{\partial x} \frac{\partial u}{\partial y}+\frac{\partial v}{\partial x} \frac{\partial v}{\partial y}\right)\right\} .
\end{aligned}
$$

t The restrictions imposed by (3) do not preclude the possibility that a second order approximation to a simple fluid of the form (1) could hold with $x_{1}<0$ and $\alpha_{1}+\alpha_{2} \neq 0$. Of course it precludes an exact model which obeys the Clausius-Duhem inequality in all motions with $\alpha_{1}<0$ and $\alpha_{1}+\alpha_{2} \neq 0$. We refer the reader to [8] and [9] for further details. 
The usual boundary layer arguments that $u, \frac{\partial u}{\partial x}, \frac{\partial^{2} u}{\partial x^{2}}, \frac{\partial p}{\partial x}$ be $O(1)$ and $y$ be $O(\delta)$ lead to (cf. [2])

$$
\begin{aligned}
u \frac{\partial u}{\partial x}+\frac{\partial u}{\partial y}= & -\frac{1}{\rho} \frac{\partial p}{\partial x}+v \frac{\partial^{2} u}{\partial y^{2}}+\frac{\alpha_{1}}{\rho}\left[\frac{\partial u}{\partial y} \frac{\partial^{2} v}{\partial y^{2}}+v \frac{\partial^{3} u}{\partial y^{3}}+\frac{\partial}{\partial x}\left(u \frac{\partial^{2} u}{\partial y^{2}}\right)+2 \frac{\partial}{\partial x}\left(\frac{\partial u}{\partial y}\right)^{2}\right] \\
& -\frac{1}{\rho} \frac{\partial p}{\partial y}+2 \frac{\alpha_{1}}{\rho} \frac{\partial}{\partial y}\left\{\left(\frac{\partial u}{\partial y}\right)^{2}\right\}=0
\end{aligned}
$$

where terms of $O(\delta)$ have been neglected.

Defining

$$
p^{*}=p-2 \alpha_{1}\left(\frac{\partial u}{\partial y}\right)^{2}
$$

equations (8a) and (8b) can be rewritten as

$$
\begin{aligned}
u \frac{\partial u}{\partial x}+v \frac{\partial u}{\partial y}= & -\frac{1}{\rho} \frac{\partial p^{*}}{\partial x}+\frac{\mu}{\rho} \frac{\partial^{2} u}{\partial y^{2}}+\frac{\alpha_{1}}{\rho}\left[\frac{\partial u}{\partial y} \frac{\partial^{2} v}{\partial y^{2}}+v \frac{\partial^{3} u}{\partial y^{3}}+\frac{\partial}{\partial x}\left(u \frac{\partial^{2} u}{\partial y^{2}}\right)\right] \\
& -\frac{1}{\rho} \frac{\partial p^{*}}{\partial y}=0
\end{aligned}
$$

both $v(=\mu / \rho)$ and $\alpha_{1} / \rho$ being $\mathrm{O}\left(\delta^{2}\right), \delta$ being the boundary layer thickness. It follows from (9b) that $p^{*}=p^{*}(x)$ and consequently

$$
U_{e} \frac{\partial U_{e}}{\partial x}=-\frac{1}{\rho} \frac{\partial p^{*}}{\partial x}
$$

where $U_{e}$ is the velocity of the main flow outside the boundary layer. Equation (9) can be non-dimensionalized in the usual manner by scaling the velocities with respect to $U_{e}$ and the lengths $x$ and $y$ with respect to a characteristic length $L$. The Reynolds number can also be eliminated from the above equation by the use of a further transformation which scales velocities with the square root of the Reynolds number.

Thus, we obtain the following non-dimensional forms of the continuity and boundary layer equations (the bar quantities representing the non-dimensional forms)

$$
\begin{gathered}
\frac{\partial \bar{u}}{\partial \bar{x}}+\frac{\partial \bar{v}}{\partial \bar{y}}=0 \\
\bar{u} \frac{\partial \bar{u}}{\partial \bar{x}}+\bar{v} \frac{\partial \bar{u}}{\partial \bar{y}}=\bar{U}_{e} \frac{\mathrm{d} \bar{U}_{e}}{\mathrm{~d} \bar{x}}+\frac{\partial^{2} \bar{u}}{\partial \bar{y}^{2}}+\varepsilon\left[\frac{\partial}{\partial \bar{x}}\left(\bar{u} \frac{\partial^{2} \bar{u}}{\partial \bar{y}^{2}}\right)+\frac{\partial \bar{u}}{\partial \bar{y}} \frac{\partial^{2} \bar{v}}{\partial \bar{y}^{2}}+\bar{v} \frac{\partial^{3} \bar{u}}{\partial \bar{y}^{3}}\right]
\end{gathered}
$$

where $\varepsilon=\frac{\alpha_{1}}{\rho L^{2}(\sqrt{R})}, R$ being the Reynolds number.

The appropriate boundary conditions are

$$
\begin{array}{ccc}
\bar{u}=\bar{v}=0 & \text { at } & \bar{y}=0, \\
\bar{u} \rightarrow \bar{U}_{e}(\bar{x}) & \text { as } & \bar{y} \rightarrow \infty .
\end{array}
$$


The stream funclion $\bar{\psi}$ defined through

$$
\bar{u}=\frac{\hat{\psi} \bar{\psi}}{\hat{\imath} \bar{y}}, t=-\frac{\hat{\psi} \bar{\psi}}{\hat{\imath} \bar{x}}
$$

satisfies equation (11) automatically. Equation (12) reduces to

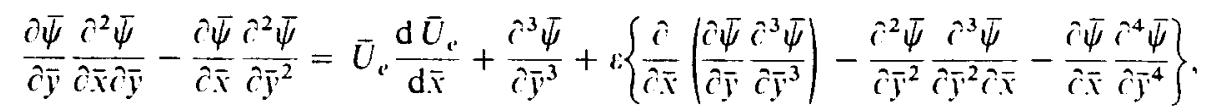

and the boundary conditions are

$$
\begin{aligned}
& \frac{\partial \bar{\psi}}{\partial \bar{x}}=\frac{\partial \bar{\psi}}{\partial \bar{y}}=0 \text { at } \bar{y}=0, \\
& \frac{\partial \bar{\psi}}{\partial \bar{y}} \rightarrow \bar{U}_{e}(\bar{x}) \text { as } \bar{y} \rightarrow x .
\end{aligned}
$$

FALKNER - SKAN FLOW

In this section we study the boundary layer flow of an incompressible second grade fluid past a wedge placed symmetrically with respect to the flow direction. Included as special cases are the flow past a flat plate and the flow near a stagnation point.

We shall assume that the stream function $\bar{\psi}$ can be expanded in a power series in $:$ (cf. Beard and Walters [3])

$$
\bar{\psi}=\bar{\psi}_{0}(\bar{x}, \bar{y})+\varepsilon \bar{\psi}_{1}(\bar{x}, \bar{y})+\cdots+\varepsilon^{n} \bar{\psi}_{n}(\bar{x}, \bar{y})+\cdots .
$$

On substituting (17) into (15) and (16a) and (16b) and equating powers of $x$ one obtains the following equations at zero-th and first order, respectively:

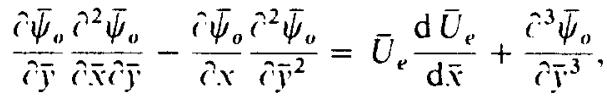

$$
\begin{aligned}
& \frac{\hat{\imath} \bar{\psi}_{0}}{\hat{C} \bar{X}}=\frac{\hat{\imath} \bar{\psi}_{0}}{\hat{\imath} \bar{y}}=0 \text { at } \bar{y}=0 \text {, } \\
& \frac{\partial \bar{\psi}_{o}}{i \bar{y}} \rightarrow \bar{U}_{c}(\bar{x}) \text { as } \bar{y} \rightarrow \infty \text {. }
\end{aligned}
$$

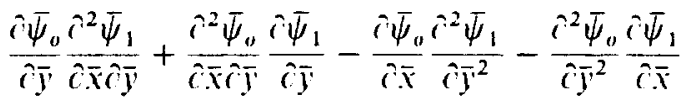

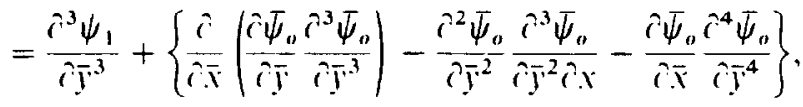

$$
\begin{aligned}
& \frac{\partial \bar{\psi}_{1}}{\partial \bar{x}}=\frac{\hat{c} \bar{\psi}_{1}}{\partial \bar{y}}=0 \text { at } \bar{y}=0, \\
& \frac{\hat{\imath} \bar{\psi}_{1}}{i \bar{y}} \rightarrow 0 \text { as } \quad \bar{y} \rightarrow \infty \text {. }
\end{aligned}
$$

Introducing the similarity transformation

$$
\eta=\sqrt{\frac{m+1}{2}} \frac{\bar{y}}{\bar{x}\left(\frac{1-m}{2}\right)}
$$


and

$$
f_{0}(\eta)=\sqrt{\frac{m+1}{2}} \frac{\bar{\psi}_{o}}{\bar{x}\left(\frac{1+m}{2}\right)},
$$

we can reduce equation (10) to the following ordinary differential equation

$$
f_{o}^{\prime \prime \prime}+f_{o} f_{o}^{\prime \prime}+\frac{2 m}{m+1}\left(1-f_{o}^{\prime 2}\right)=0
$$

The appropriate boundary conditions are

$$
\begin{gathered}
f_{o}(0)=f^{\prime}(0)=0 \text { at } \eta=0, \\
f_{o}^{\prime}(\infty) \rightarrow 1 \text { as } \eta \rightarrow \infty,
\end{gathered}
$$

provided

$$
\bar{U}_{e}=\bar{x}^{m}
$$

In equation (24), the primes are differentiations with respect to $\eta$. Equation (24) is the classical Falkner-Skan equation for boundary layer flow of Newtonian fluids with main stream velocity given by equation (26).

To obtain the solution of the first-order equation, we employ the following transformation in addition to the transformations (22) and (23)

$$
f_{1}(\eta)=\frac{\bar{\psi}_{1}}{\left.\bar{x} \mid \frac{3 m-1}{2}\right)} \sqrt{\frac{m+1}{2}} .
$$

In this case the equation (20) becomes

$$
\begin{aligned}
& \frac{m+1}{2} f_{1}^{\prime \prime \prime}+\frac{m+1}{2} f_{o} f_{1}^{\prime}-(3 m-1) f_{o}^{\prime} f_{1}^{\prime}+\frac{3 m-1}{2} f_{o}^{\prime \prime} f_{1} \\
& +\left\{(3 m-1) f_{o}^{\prime} f_{o}^{\prime \prime \prime}-\frac{3 m-1}{2}\left(f_{o}^{\prime \prime}\right)^{2}-\frac{m+1}{2} f_{o} f_{o}^{\prime \prime}\right\}=0 .
\end{aligned}
$$

The appropriate boundary conditions are

$$
f_{1}(0)=f_{1}^{\prime}(0)=0, \quad f_{1}^{\prime}(\eta) \rightarrow 0 \quad \text { as } \quad \eta \rightarrow \infty .
$$

SOLUTIONS AND DISCUSSIONS

Numerical solutions for the zero-th order perturbation, namely equations (24) and (26), are well known (cf. Moore [10]). The initial slopes, $f_{o}^{\prime \prime}(0)$, for different positive values of $\beta$ 's are given in Table 1.

We next solve (28), subject to the boundary conditions (29). The equation (28) is first written as a first-order system. The derivatives are then approximated by centered-difference gradients and averages centered at the midpoints of the net defined by

$$
\eta_{o}=0, \eta_{j}=\eta_{j-1}+\mathrm{h}_{j}, \mathrm{j}=1,2, \ldots, \mathrm{J} \quad \eta_{J}=\eta_{\infty}
$$

A non-uniform grid $h_{j}$ is defined through

$$
h_{j}=k h_{j-1}
$$


Table 1. $f_{n}^{\prime \prime}(0)$ for various $\beta$ s $\left(\beta=\frac{2 m}{m+1}\right)$

\begin{tabular}{cccc}
\hline$\beta$ & $f_{o}^{\prime \prime}(0)$ & $\beta$ & $f_{o}^{\prime \prime \prime}(0)$ \\
\hline 0.05 & 0.531130 & 0.60 & 0.995836 \\
0.10 & 0.587035 & 0.80 & 0.120268 \\
0.20 & 0.686708 & 1.00 & 1.232585 \\
0.30 & 0.774755 & 1.20 & 1.335722 \\
0.40 & 0.854421 & 1.60 & 1.521514 \\
0.50 & 0.927680 & & \\
\hline
\end{tabular}

where the ratio of adjacent intervals, $k$, is a constant is employed. The distance from the surface to the $j$ th station is given by:

$$
\eta_{j}=h_{j} \begin{gathered}
k^{j}-1 \\
k-1
\end{gathered}, j=1,2, \ldots, J
$$

Linearization is achieved by the method of quasilinearization and the resulting system of algebraic equations are then solved by a block-tridiagonal factorization technique (cf. $\mathrm{Na}[11])$. The method is unconditionally stable and second-order accurate.

Table 2 gives the initial slopes of the first-order solution, $f_{1}^{\prime \prime}(0)$, for the same $\beta$ 's as in Table 2 , and plotted in Fig. 1.

Equation (34) indicates that when $\beta$ is small and $\varepsilon$ is negative, the skin friction decreases with increasing $|\varepsilon|$. However, if $\beta$ is large and $\varepsilon$ is negative, the skin friction increases with increasing $|\varepsilon|$. Such a reversal is the result of the change of sign of $f_{1}^{\prime \prime}(0)$ shown in Fig. 1 . On the other hand, if $\varepsilon$ is positive, the skin friction increases with $\varepsilon$ for small $\beta$ and decreases with $\varepsilon$ for large $\beta$.

Figures 2 and 3 show the variation of $f_{1}^{\prime}(\eta)$ with respect to $\eta$ for $\beta=0.1$ and $\beta=1$, respectively. The curves, as can be seen, are qualitatively different.

Table 2. $f ;(0)$ for various $\beta$ s

\begin{tabular}{crcc}
\hline$\beta$ & \multicolumn{1}{c}{$l_{i}^{\prime \prime}(0)$} & $\beta$ & \multicolumn{1}{c}{$f_{1}^{\prime \prime}(0)$} \\
\hline 0.05 & 0.82139 & 0.60 & -0.34086 \\
0.10 & 0.52959 & 0.80 & -0.71638 \\
0.20 & 0.30086 & 1.00 & -1.13902 \\
0.30 & -0.14178 & 1.20 & -1.60638 \\
0.40 & -0.01118 & 1.60 & -2.66413 \\
0.50 & -0.17077 & & \\
\hline
\end{tabular}

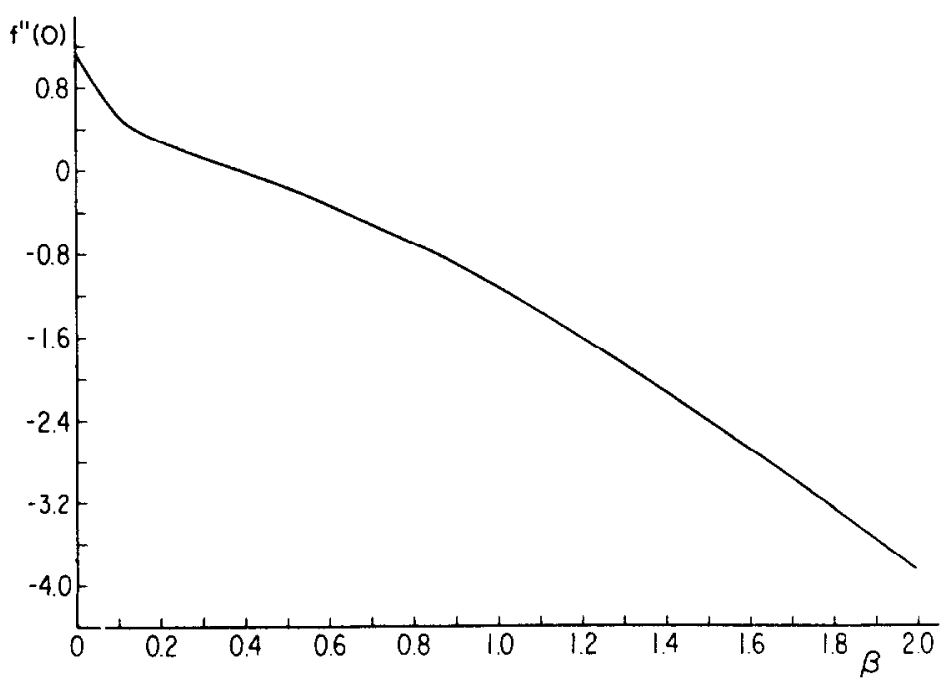

Fig. 1. $f^{\prime \prime}(0)$ vs $\beta$. 


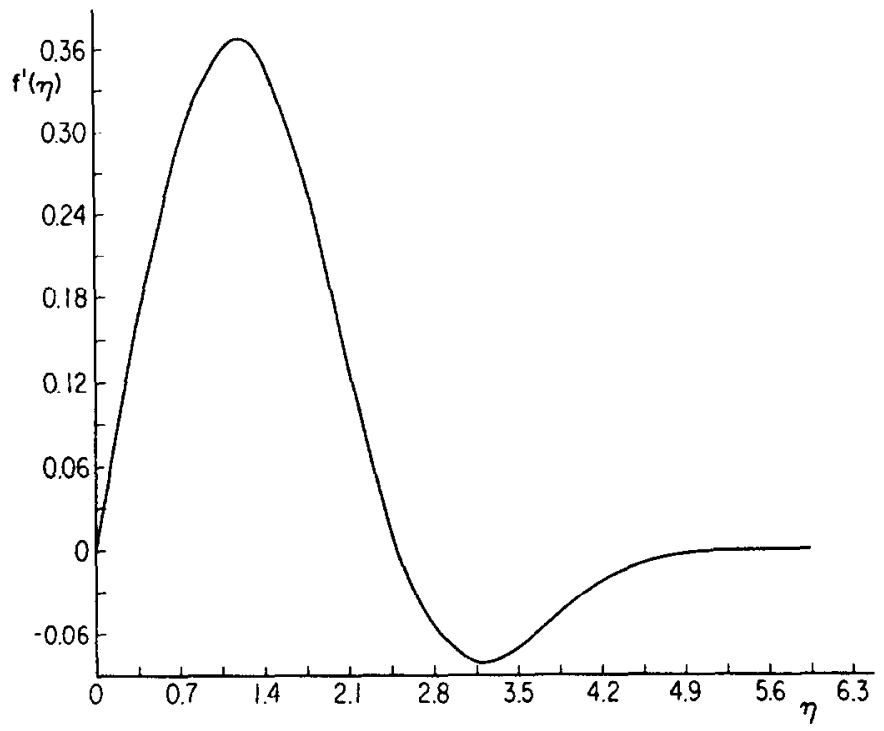

Fig. 2. $f(1)$ vs $\eta$

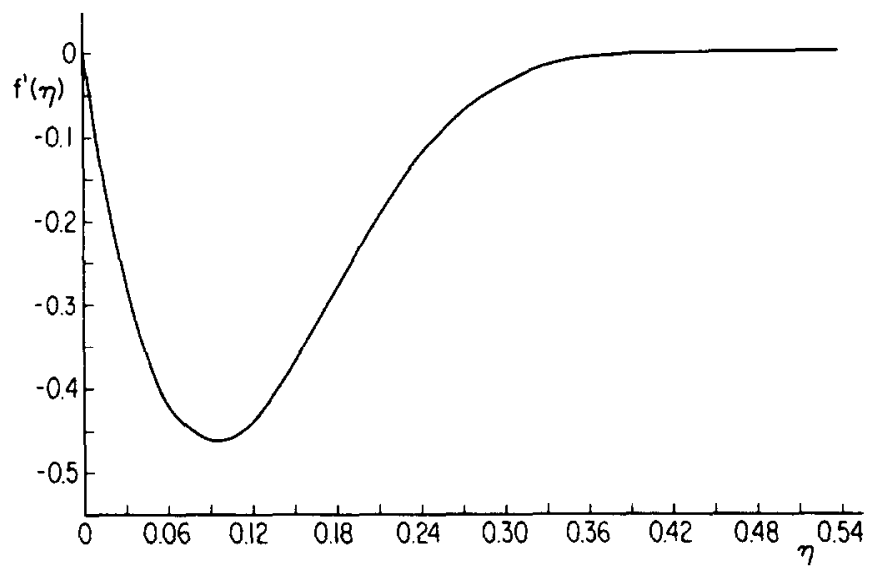

Fig. 3. $f^{\prime \prime}(\eta)$ vs $\eta$.

\section{REFERENCES}

1. A. C. Srivatsava, The flow of a non-Newtonian liquid near a stagnation point, Z. angen: Math. Phys. 9, 8-84 (1958).

2. G. Rajeswari and S. L. Rathna, Flow of a particular class of non-Newtonian visco-elastic and visco-inelastic fluids near a stagnation point, $Z$. angew. Math. Phys. 13, 43-57 (1962).

3. D. W. Beard and K. Walters, Elastico-viscous boundary layer flows, Proc. Camb. phil Soc. math. phys. Sci. 667-674 (1964).

4. K. R. Rajagopal. A. S. Gupta and A. S. Wineman, On a boundary layer theory for non-Newtonian fluids, Le't Appl. Sci. Engng, 18, 875-88.3 (1980).

5. C. Truesdell and W. Noll. The non-linear field theories of mechanics, Handh. Phys. IIt/3, (1965).

6. R. S. Rivlin and J. L. Ericksen. Stress deformation relation for isotropic materials, J. rat. Mech. Analysis 4. $323-425(1955)$.

7. B. D. Coleman and W. Noll, An approximation theorem for functionals with applications in continuum mechanics, Archs ration Mech. Anulysis 6. 355-370 (1960).

8. J. E. Dunn and R. L. Fosdick. Thermodynamics, stibility and boundedness of fluids of complexity 2 and fluids of second grade, Archs Ration. Mech. Analysis 3, 191-252 (1974).

9. R. L. Fosdick and K. R. Rajagopal, Anomalous features in the model of "second order fluids", Archs ration. Mech. Analisis 70. 145-152 (1979).

10. F. K. Moore. Theory of Laminar Flows. Princeton University Press, NJ (1964).

11. T. Y. Na. Computational Methods in Engineering: Boundary Valuc Problems. Academic Press. New York (1979). 
Résumé:

On établit des solutions non similaries pour l'écoulement de couche limite d'un fluide homogene incompressible du second ordre devant un coin placé symétriquement par rapport à la direction de l'écoulement. On discute la variation du frottement de peau par rapport aux paramètres non Newtoniens.

\section{Zus ammen fassung:}

Fuer die Grenzschichtstroemung einer homogenen und inkompressiblen Fluessigkeit zweiten grades veber einen in Bezug auf die Stroemungsichtung symmetrisch angeordineten Keil werden nichtaehnliche Loesungen aufgestellt. Die Veraenderung der Oberflaechenreibung bezueglich der nichtnewtonschen Parameter wird diskutiert. 